\title{
Vicente Díaz, Martha Roxana. 2018. El sentido de lo alternativo en la economía solidaria: La experiencia de Yomol A'tel. Cuadernos Deusto de Derechos Humanos 92. Bilbao: Universidad de Deusto
}

doi: http://dx.doi.org/10.18543/djhr.2117

En los tiempos actuales donde impera una crisis en las dimensiones económicas, políticas, sociales y ambientales, se requiere en mayor medida una consciencia colectiva sobre la forma de consumir y producir. Preguntas como: ¿de dónde proviene lo que la sociedad consume? ¿Cuáles son las condiciones bajo las que se producen los productos que se deciden consumir en los hogares? ¿Quiénes participan como actores en todo el proceso de la cadena de producción, suministro y consumo? ¿Son respetados los derechos humanos de quienes intervienen en este proceso, especialmente en la etapa de producción?, son necesarias y relevantes para reconocer las distintas formas en las que se violan los derechos humanos de las personas.

Muchas historias explican el modo en que son violados los derechos humanos de las personas en procesos como la producción del café y el impacto para los productores (Morales de la Crus 2018), así como la explotación de personas en un sector como el cultivo de café donde $36 \%$ del territorio en el sur de México (el estado de Chiapas) se dedica específicamente a dicho cultivo (Red Nacional de Jornaleros y Jornaleras Agrícolas 2019). Algunos otros ejemplos son la violación de derechos humanos de las personas que consiguen coltán en países como la República del Congo, conocido como el mineral de sangre y que se utiliza en la fabricación de productos tecnológicos (Blay, 2016), o la deshumanización de las personas que trabajan en maquiladoras de fast fashion (Vlasich 2020) así como el impacto ambiental de esta moda (Villemain 2019). Lo anterior refleja las consecuencias que se generan cuando no existe reflexión sobre las propias elecciones en el consumo, y en las que se olvida anteponer el bienestar común y la dignidad humana. Al elegir opciones de comercio justo, es decir, aquellas que respetan el trabajo de productores, se permite conservar empleos, aumentar el ingreso de comunidades y promover que los problemas sociales disminuyan, debido a que se elige el énfasis en dicho bienestar. Muchas de las iniciativas que promueven este tipo de actividades responsables, conscientes, de cooperación y ayuda mutua se identifican como Economía Social y Solidaria (ESS). 
En un mundo donde la desigualdad y la pobreza persisten y cada día se intensifican más, es importante considerar y revisar ejemplos de iniciativas que busquen mitigar estos problemas sociales, como el caso de las empresas de la ESS. A nivel mundial, los problemas como la pobreza o la desigualdad son parte de los objetivos principales que se buscan atacar. Los Objetivos de Desarrollo Sostenible dan cuenta de ello. A través del objetivo uno -terminar con la pobreza- y el objetivo diez -terminar con todas las formas de desigualdad y discriminaciónse plantea que es un imperativo para todas las sociedades seguir lineamientos a nivel local, regional, nacional e internacional que puedan promover oportunidades para todas las personas, específicamente aquellos sectores de la población que sistemáticamente han sido olvidados, discriminados, excluidos o anulados. Aquellos sectores de la población que, dada la violencia no sólo estructural sino simbólica, han permanecido en aquellos espacios sin voz y que debieran ser escuchados.

La investigación de Martha R. Vicente en El sentido de lo alternativo en la economía solidaria: La experiencia de Yomol A'tel brinda voz a esas personas olvidadas en una doble dirección. Por un lado, analiza el caso de estudio de una empresa social y solidaria en México (Yomol $A^{\prime}$ tel) que ha trabajado con un grupo excluido y olvidado en aras de brindar oportunidades a esta comunidad. Por otro lado, el ejercicio que realiza la investigación brinda voz a las personas de dicho grupo que integran la organización. Desde la recuperación de la narrativa de sus percepciones, analizar el impacto que genera el proyecto de Yomol A'tel en términos sociales y de sus propias vidas.

A través de una investigación conformada en cinco secciones, la autora realiza un estudio sobre el impacto de la empresa social y solidaria de Yomol A'tel en el sur de México, en las comunidades de Chiapas. Este ejercicio lo realiza basado en un estudio de tipo cualitativo en el que, desde un proceso narrativo, introduce los aspectos positivos que la empresa ha generado en el territorio, además de contar con los desafíos que genera luego de más de una década de creación de la empresa.

Al leer esta investigación, tenemos la oportunidad de entender distintos aspectos sumamente complejos que se reflejan en la sociedad en las palabras de la autora. A continuación se presenta una breve recapitulación de los capítulos que conforman la investigación.

En el capítulo uno la autora establece los elementos que identifican las circunstancias bajo las que se crea Yomol A'tel, la historia de la organización, y la conceptualización que tienen sobre la misma. Asimismo, profundiza en la estrategia que adquiere la empresa para 
legitimar su proyecto en la región y, con ello, brindar continuidad y acompañamiento durante varios años a las personas que forman parte de ella. La empresa nace con el objetivo de brindar oportunidades en una región que históricamente ha vivido en condiciones de pobreza y desigualdad. El proyecto, guiado bajo los principios jesuitas y basado en la «inculturación», ha promovido oportunidades de empleo para los productores de la región, conformando una red que enfrenta el principal problema de la producción del café: el coyotaje. Este fenómeno ocurre cuando los intermediarios compran el café por un precio muy bajo y lo revenden en precios altos, obteniendo un alto margen de ganancia. Esto promueve que los productores no obtengan los ingresos suficientes y sigan viviendo condiciones de precariedad. De este modo, la estrategia de Yomol A'tel pretende promover el comercio justo y escuchar a los productores de la región, contribuyendo al fortalecimiento de capacidades de dichos productores para la mejora de la calidad de los mismos.

En el capítulo dos, establece el marco analítico desde el cual se observará el caso de análisis, es decir, las líneas que servirán como guía para entender el estudio de caso. En primer lugar, realiza una revisión del concepto de la ESS a nivel internacional y luego considerando el caso específico de México. Después, genera un análisis sobre el debate con relación a las empresas sociales y solidarias en el país, así como los principales desafíos éticos que tienen estas empresas desde una perspectiva de la ESS. Finalmente, desde un marco basado en el pensamiento descolonial, analiza el rol de las empresas de ESS como un agente de transformación social, entendiendo el concepto de alternativo como aquello que permite esos cambios desde la toma de consciencia.

En el capítulo tres, la autora detalla la metodología que siguió para realizar esta investigación. En este apartado habla de la forma en que la etnografía es una forma de narrar la realidad, de comunicar. Ella elige el relato como aproximación para narrar su experiencia. La inquietud de profundizar en el fenómeno de la empresa Yomol A'tel surge a través de su trabajo previo con comunidades indígenas. De esta forma, el interés que mantiene es descubrir y entrecruzar las distintas perspectivas que existen sobre el fenómeno que, en este caso, son las percepciones que tienen sobre la organización. Esto lo llevó a cabo a través de la participación en el proceso de sistematización que realizaron en la organización en su segunda versión en 2014.

La sistematización se considera un método innovador que se vincula con las experiencias de educación popular en América Latina, 
y lo que busca es generar un relato que describa la realidad, que reconstruya a través de los distintos relatos de la diversidad de actores y fuentes el macro relato que incluye a todas las voces. En el caso de Yomol A'tel, se había realizado previamente una sistematización para reconocer los desafíos que enfrentaba la organización a finales de la década de 2010. Martha R. Vicente participa en una segunda versión con el objetivo de identificar los desafíos luego de la crisis del café. En esta ocasión se realizaron 25 entrevistas y 11 grupos focales. Con esta información, la autora realiza una discriminación de los relatos que más correspondan a los objetivos de la investigación. Con ello, se eligen las dimensiones críticas en las siguientes temáticas: Yomol A'tel como empresa social y solidaria; la relación del proyecto con la idea de la buena vida tseltal o la idea del lekil kuxlejalil; la dinámica de las relaciones de poder dentro y fuera de la organización; los espacios de crítica y reflexión ética que se generan en la organización, así como la forma en que incide el proyecto en la vida de las personas que forman parte de la organización (tanto en el aspecto personal, como el social).

El cuarto capítulo es el análisis de la experiencia en la organización Yomol A'tel. Se lleva a cabo en dos apartados. El primero se refiere al análisis de los relatos personales que seleccionó la autora para la investigación, donde se observa que la organización es un camino de aprendizajes para todos los que colaboran en el proyecto, además de lo que llama la autora un «dispositivo de inserción laboral», refiriéndose a las oportunidades de los productores y los hijos de los mismos para encontrar un trabajo y así evitar la migración y abandono de las familias y comunidades. Sin embargo, también hace una reflexión sobre las contradicciones en las dinámicas que se viven en la organización, sobre todo, con relación a la participación en la toma de decisiones en el proyecto.

El segundo apartado es el análisis de la información obtenido de los grupos focales en los que participaron los socios productores de la cooperativa de café. En este aspecto, los productores externan la necesidad de acompañamiento técnico por parte de Yomol A'tel, luego de la plaga de roya en 2014. Asimismo, se muestra una fuerte vinculación a la dimensión espiritual en todos los aspectos de la vida comunitaria. Otro de los aspectos que se menciona es la conciencia crítica que adquieren las mujeres participantes en el proyecto, empoderando a este grupo que estructuralmente ha sido uno de los más desfavorecidos. Finalmente, menciona el debate entre la relevancia del aspecto económico frente al bienestar a largo plazo que el proyecto puede brindar. 
El capítulo cinco recupera las reflexiones por parte de la investigadora con relación a las experiencias del proceso de dicha investigación. En la primera parte hace un análisis de lo alternativo desde la experiencia con Yomol A'tel. Asimismo, profundiza en la importancia de la sistematización para construir lo alternativo y, con ello, entender la relevancia de lo que la organización genera en términos de transformación social.

Respecto a la relación con lo alternativo, explica que Yomol A'tel ha logrado generar una cadena de valor que permite un precio justo para los productores que forman parte del proyecto. Así, Yomol A'tel es una empresa social y solidaria porque pone en el centro a las personas, y deja al servicio de la comunidad tseltal el trabajo que se realiza en toda la cadena productiva. De la misma forma, busca que pierdan fuerza en el territorio la red de intermediarios que desvalorizan el trabajo de la comunidad y perpetúan la situación de precariedad y marginalización en la región.

Asimismo, el proyecto mantiene relación con la idea de la buena vida tseltal o el lekil kuxlejalil, el cual ha formado parte del proceso en todas sus dimensiones, y brinda una especie de resistencia reguladora en el proyecto. Lo anterior implica que al proyecto lo atraviesa un ritmo propio, basado en los valores y creencias de la vida tseltal, que orienta el camino del mismo a través de una constante reflexión ética. El proyecto de Yomol A'tel trabaja con el concepto de lekil kuxlejalil, que orienta el proyecto por los deseos del corazón de la comunidad tseltal. Para la autora, la buena vida tseltal implica «tener el corazón en casa -nacal o'tanil» que significa estar tranquilo, sereno y con una sensación de vinculación al entorno. Cuando eso se rompe, es que el equilibrio se ha roto y es importante buscar la vinculación a través de procesos como la oración, el diálogo, con la fiesta o la danza. Así, esta buena vida es un modo de caminar y una ética de la vida.

Con respecto a los procesos de transformación social, la autora reflexiona el rol de la organización como respuesta integral a las condiciones de marginalización que existen en la sociedad, brindando oportunidades en términos de empleo. Sin embargo, la escuela de café que crea Yomol A'tel dentro del proyecto genera otras oportunidades, entre las que destaca el permitir la consciencia de los tseltales de su realidad y de buscar generar un cambio para ellos mismos. Ello lo menciona a través de aspectos como la tseltalización del proyecto, es decir, que el proyecto sea liderado por personas de la comunidad de forma paulatina, alcanzando la autogestión y autonomía por parte de Yomol A'tel. 
Finalmente, se refiere también a la forma en la que las investigaciones generan efecto en las personas con relación a procesos de transformación social. Cuando se logran cambios en comportamientos y existe una actitud de consciencia ante la realidad, es que existe un efecto desde el ámbito de la investigación. Establece que la investigación es un proceso de construcción colectiva, en donde se generan espacios de reflexión y donde las personas tienen la oportunidad de descubrirse y construirse. Concluye, con la reflexión de que su investigación se ha realizado con dicho objetivo: promover los espacios de reflexión para la toma de consciencia de los participantes.

La investigación plantea, en suma, la oportunidad de observar los procesos de reflexión y aprendizaje que algunas empresas de la ESS llevan a cabo con el objetivo de mejorar sus procesos y guiarse bajo los principios de las mismas, principalmente el principio de igualdad. Analizar el caso del proyecto Yomol A'tel, permite observar la realidad de la ESS en la sociedad mexicana de manera indirecta, y plantear los cuestionamientos y necesidades que el caso mexicano exige, como la adaptación del marco jurídico de la ESS en el país, así como el apoyo por parte de distintos actores para visibilizar al sector social en México. Investigaciones como la que realiza Vicente son necesarias, para mostrar la importancia de proyectos como Yomol A'tel a nivel nacional y difundir este tipo de buenas prácticas. Sólo de esta forma la sociedad podrá ser más consciente de lo que consume y elegirá de forma asertiva, reconociendo el proceso en la cadena de producción existente para los productos que se eligen.

\section{Bibliografía}

Blay, Blanca. 2016. "La cara más oscura de la tecnología», El diario. Acceso el 17 de septiembre de 2020. https://www.eldiario.es/catalunya/coltancongo-cara-oscura-tecnologia-movil_1_4139886.html

Morales-de la Cruz, Fernando. 2018. «La crisis del café es una oportunidad única para poner fin a la explotación», El economista, Acceso el 17 de septiembre de 2020. https://www.eleconomista.com.mx/opinion/Lacrisis-del-cafe-es-una-oportunidad-unica-para-poner-fin-a-la-explotacion20181105-0036.html

Red Nacional de Jornaleros y Jornaleras Agrícolas. 2019. Violación de derechos de las y los jornaleros agrícolas en México. Primer Informe. Acceso el 17 de septiembre de 2020. http://cecig.org.mx/wp-content/uploads/2019/03/ INFORME_RNJJA_2019.pdf 
Villemain, Cyril. 2019. "El costo ambiental de estar a la moda» Noticias ONU. Acceso el 20 de septiembre de 2020. https://news.un.org/es/ story/2019/04/1454161

Vlasich, Valeria. 2020. «Fast fashion: el caso de Bangladesh», Este país. Acceso el 17 de septiembre de 2020. https://estepais.com/ambiente/fast-fashionel-caso-de-bangladesh/

Raquel Ortiz-Ledesma Universidad de Deusto 


\section{Copyright}

Deusto Journal of Human Rights / Revista Deusto de Derechos Humanos is an Open Access journal; which means that it is free for full and immediate access, reading, search, download, distribution, and reuse in any medium only for non-commercial purposes and in accordance with any applicable copyright legislation, without prior permission from the copyright holder (University of Deusto) or the author; provided the original work and publication source are properly cited (Issue number, year, pages and DOI if applicable) and any changes to the original are clearly indicated. Any other use of its content in any medium or format, now known or developed in the future, requires prior written permission of the copyright holder.

\section{Derechos de autoría}

Deusto Journal of Human Rights / Revista Deusto de Derechos Humanos es una revista de Acceso Abierto; lo que significa que es de libre acceso en su integridad inmediatamente después de la publicación de cada número. Se permite su lectura, la búsqueda, descarga, distribución y reutilización en cualquier tipo de soporte sólo para fines no comerciales y según lo previsto por la ley; sin la previa autorización de la Editorial (Universidad de Deusto) o la persona autora, siempre que la obra original sea debidamente citada (número, año, páginas y DOI si procede) y cualquier cambio en el original esté claramente indicado. Cualquier otro uso de su contenido en cualquier medio o formato, ahora conocido o desarrollado en el futuro, requiere el permiso previo por escrito de la persona titular de los derechos de autoría. 